\title{
Combination Therapy with PIK3R3-siRNA and EGFR-TKI Erlotinib Synergistically Suppresses Glioblastoma Cell Growth In Vitro
}

\author{
Razieh Amini ${ }^{1}$, Hadi Karami ${ }^{1 *}$, Mohammad Bayat ${ }^{2}$
}

\begin{abstract}
Background: Up-regulation of PIK3R3 (Phosphoinositide-3-Kinase Regulatory Subunit 3), the regulatory subunit of PI3K is correlated with the drug resistance of the glioblastoma cells. In the present study, the effect of $P I K 3 R 3$ siRNA on erlotinib sensitivity of the U373-MG glioblastoma cells was explored. Methods: After PIK3R3 siRNA transfection, the expression of $P I K 3 R 3$ mRNA was measured using RT-qPCR. Trypan blue exclusion assay was used to explore the effect of $P I K 3 R 3$ siRNA on cell proliferation. The effects of $P I K 3 R 3$ siRNA and erlotinib, alone and in combination, on cell survival and apoptosis were measured using MTT assay and ELISA cell death assay, respectively. Results: Our data showed that PIK3R3 siRNA markedly suppressed the expression of PIK3R3 in a time dependent way, inhibited the proliferation of the U373-MG cells and triggered apoptosis ( $<<0.05$, relative to blank control). Pretreatment with $P I K 3 R 3$ siRNA synergistically decreased the cell survival rate and lowered the $\mathrm{IC}_{50}$ of erlotinib. Moreover, $P I K 3 R 3$ siRNA markedly enhanced the apoptotic effect of erlotinib. Conclusions: Our data propose that suppression of $P I K 3 R 3$ can effectively triggers apoptosis and enhances the sensitivity of the glioblastoma cells to EGFR-TKI erlotinib. Thus, $P I K 3 R 3$ can be a potential therapeutic target in glioblastoma patients.
\end{abstract}

Keywords: Erlotinib- Glioblastoma- PIK3R3- PI3K- SiRNA

Asian Pac J Cancer Prev, 22 (12), 3993-4000

\section{Introduction}

Glioblastoma multiform is the most aggressive form of glioma that accounts for over $50 \%$ of brain tumors (García-Claver et al., 2013; Alamdari-Palangi et al., 2020a). Common treatments for glioblastoma include surgery, radiotherapy, and chemotherapy with temozolomide, bis-chloroethylnitrosourea and carmustine (Ruano et al., 2008; García-Claver et al., 2013). Despite all medical efforts in recent years, glioma indicates a high resistance to treatment, and patients show a low rate of survival, which underlines the need for effective therapies (Mellinghoff et al., 2005; Ruano et al., 2008; García-Claver et al., 2013; Alamdari-Palangi et al., 2020b). One of the important approaches for treatment that is being investigated is selective targeting of certain molecule and signaling pathways that are involved in the proliferation of glioblastoma cells (Ruano et al., 2008).

The epidermal growth factor receptor (EGFR) is a member of the HER family receptors that is involved in different cell functions including proliferation and differentiation (Amri et al., 2019a; Amri et al., 2019b; Amri et al., 2021). Over-expression of EGFR gene has been extensively observed in different types of human malignant cells, including glioma. In these tumors, EGFR signaling can be restrained at the level of the receptor or through down-stream signaling mediators like PI3K/ protein kinase B (PKB)/AKT pathway (Paul et al., 2013; Oprita et al., 2021). The Phosphoinositide 3-kinase (PI3K) pathway is implicated in a wide variety of cancers. It also seems to be associated with the phenotype of glioblastoma cells including survival, invasion, proliferation, cell growth and resistance to treatment (Ruano et al., 2008; Halatsch et al., 2009). PI3K is an intracellular signal transmitter which is activated by several tyrosine kinase receptors and produces a secondary messenger in the membrane (Vredenburgh et al., 2007; Kreisl et al., 2009). Amplification or mutations of PIK3R3 (Phosphoinositide3-Kinase Regulatory Subunit 3) gene, which codes for PI3KCA protein, the regulatory subunit of PI3K, have been reported in several solid tumors (Tsao et al., 2005).

Erlotinib is a tyrosine kinase inhibitor (TKI) that exerts an inhibitory effect on EGFR receptor. It is normally used as a secondary line of treatment for brain tumors (Engelman et al., 2006; Bader et al., 2011). The results of several clinical trials performed with tyrosine kinase

${ }^{1}$ Department of Molecular Medicine and Biotechnology, Faculty of Medicine, Arak University of Medical Sciences, Arak, Iran. ${ }^{2}$ Department of Anatomy, Faculty of Medicine, Arak University of Medical Sciences, Arak, Iran. *For Correspondence: h.karami@arakmu.ac.ir 
inhibitors indicate specific mechanisms of resistance toward the action of these drugs in glioma cells (Katso et al., 2001; Luo et al., 2003; Zhang et al., 2003; Parsons, 2005). Thus understanding the molecular pathways involved in resistance to treatment with EGFR inhibitors in order to design new combinatorial therapies to help improve response is essential in glioma cells. Studies have shown that the activation of EGFR leads to a higher expression of $P I K 3 R 3$ gene in erlotinib-sensitive glioblastoma and ovarian tumors cells, in comparison with that of resistant cells (Shayesteh et al., 1999b; Galanis et al., 2005b). Moreover, it has been demonstrated that the expression of PIK3R3 gene in erlotinib-sensitive tumor cell lines treated with erlotinib is reduced, while in erlotinib-resistant cell lines the expression remains the same (Soroceanu et al., 2007; Zhang et al., 2007; García-Claver et al., 2013). These results indicate that the over-expression of PIK3R 3 can be one of the major mechanisms of resistance to erlotinib, and thus suggest $P I K 3 R 3$ as an interesting potential therapeutic target for improving the response of glioblastoma cells to erlotinib.

Small interfering RNA (siRNA) mediated gene silencing is a powerful tool to suppress the transcriptional expression of a particular gene using a synthetic doublestranded RNA molecule (Karami et al., 2013; Karami et al., 2014). The aim of this study was to investigate the effect of PIK3R3-specific siRNA on proliferation, apoptosis, and erlotinib sensitivity of the U-373 MG erlotinib-resistant human glioblastoma cells.

\section{Materials and Methods}

\section{Cell culture}

The U-373 glioblastoma cells (Pasteur Institute, Tehran, Iran) were cultured in RPMI-1640 medium (Sigma-Aldrich, St. Louis, MO, USA) supplemented with 15\% fetal bovine serum (FBS) (Sigma-Aldrich), 1\% sodium pyruvate, $1 \%$ antibiotic $(100 \mu \mathrm{g} / \mathrm{ml}$ streptomycin, $100 \mathrm{U} / \mathrm{ml}$ penicillin) (Sigma-Aldrich) and $2 \mathrm{mM}$ glutamine in a $37^{\circ} \mathrm{C}$ incubator containing $5 \% \mathrm{CO}_{2}$. The cells were passaged with an initial concentration of $1 \times 10^{5}$ cells/ $\mathrm{ml}$ and used in the exponential growth phase in all experiments.

\section{Cell transfection}

PIK3R3 and negative control (NC) siGENOME siRNAs were obtained from Dharmacon (Lafayette, CO, USA). Prior to transfection, the cells were propagated in RPMI-1640 medium without antibiotics and FBS. Transfection of siRNA (at a final concentration of 100 $\mathrm{nM}$ ) was done using Lipofectamine ${ }^{\mathrm{TM}} 2000$ transfection reagent (Invitrogen, Carlsbad, CA, USA) according to the manufacturer's protocol. In brief, siRNA and lipofectamine ( $4 \mu \mathrm{l} / \mathrm{ml}$ of transfection medium) were diluted in Opti-MEM I Reduced Serum Medium (Invitrogen) separately and incubated at room temperature for $5 \mathrm{~min}$. Next, the diluted solutions were gently mixed and incubated for another $15 \mathrm{~min}$ at $25^{\circ} \mathrm{C}$. The mixtures were then added to the culture medium. After $6 \mathrm{~h}$ incubation at $37^{\circ} \mathrm{C}$ in a humidified $\mathrm{CO}_{2}$ incubator, the complete growth medium was added to the cells.

\section{$R T-q P C R$}

At various time points after transfections, total cellular RNA was extracted by AccuZolTM reagent (Bioneer, Daedeok-gu, Daejeon, Korea) according to the manufacturer's instructions. Complementary DNA (cDNA) was synthesized from $1 \mu \mathrm{g}$ of purified total RNA by using MMLV reverse transcriptase (Promega, Madison, WI, USA) and oligo-dT primer following the manufacturer's protocols. RT-qPCR was done in the LightCycler 96 System (Roche Diagnostics GmbH, Mannhein, Germany) using SYBR Premix Ex Taq (Takara Bio, Otsu, Shiga, Japan). Each real-time PCR reaction system was: $1 \mu \mathrm{l}$ of cDNA template, $0.2 \mu \mathrm{M}$ of each primer, $12 \mu \mathrm{l}$ of SYBR green reagent, and $6 \mu \mathrm{l}$ of nucleasefree distilled water. The primer sequences were as follows: forward, 5'- AGTTCCAAATGGAATGAAGGAC-3', reverse, 5'-ACСТCСТCССТTGAАATATCC-3', for PIK3R3, forward, 5'-CTACAATGAGCGTGTG-3', and reverse, 5'- GTCTCAAACATGATCTGGGTC -3', for $\beta$-actin,. The initial denaturation step at $95^{\circ} \mathrm{C}$ for $10 \mathrm{~min}$ was followed by 45 cycles at $95^{\circ} \mathrm{C}$ for $20 \mathrm{sec}$ and $60^{\circ} \mathrm{C}$ for $1 \mathrm{~min}$. Relative PIK3R3 expression was evaluated with the $2^{-(\Delta \Delta \mathrm{Ct})}$ method (Shahverdi et al., 2020; Ashofteh et al., 2021), using $\beta$-actin as the control gene. Melting curve analysis was performed to check the specificity of the primer sequences and correctness of PCR.

\section{MTT assay}

Cell toxicity was determined by $3-(4$, 5-+Dimethylthiazol-2-yl)-2, 5-diphenyltetrazolium bromide (MTT) (Sigma-Aldrich) assay. To investigate the effect of siRNAs on the response of U-373 cells to erlotinib, the $2 \times 10^{3}$ cells/well cells were transfected with $100 \mathrm{nM}$ of either PIK3R3 siRNA or NC siRNA in 96-well plates for $6 \mathrm{~h}$. Next, different concentration of erlotinib was added to each well. Erlotinib was obtained from Sigma-Aldrich, and a stock solution was prepared in dimethyl sulfoxide (DMSO) (Sigma-Aldrich). Subsequently, Twenty-four and forty-eight hours after transfection, $20 \mu \mathrm{l}$ of $5 \mathrm{mg} / \mathrm{ml}$ MTT solution were added to each well. The cells were then incubated at $37^{\circ} \mathrm{C}$ for 4 hours and the precipitate was resuspended in DMSO $(150 \mu \mathrm{l} / \mathrm{well})$. The absorbance of each well was measured by a microplate reader (Anthos ht III, Anthos Labtec Instruments GmbH, Wals, Austria) at a wavelength of $490 \mathrm{~nm}$ with a reference at $650 \mathrm{~nm}$. The survival rate (SR) was calculated using the following equation: $\mathrm{SR}(\%)=($ A Treatment $/ \mathrm{A}$ Control $) \times 100 \%$. The concentration that produced $50 \%$ cytotoxicity $\left(\mathrm{IC}_{50}\right)$ was determined with Prism 6.01 software (GraphPad Software Inc., San Diego, CA, USA).

\section{Combination effect analysis}

The combination index (CI) analysis based on the Chou-Talalay method was performed to investigate the interaction between PIK3R3 siRNA and erlotinib (Pirayesh Islamian et al., 2016; Shahverdi et al., 2021). The results of MTT assay was converted to Fraction affected (Fa; range $0-1$; where $\mathrm{Fa}=1$ represents $0 \%$ cell survival and $\mathrm{Fa}=0$ represents $100 \%$ cell survival) and analyzed with the CompuSyn software from Combosyn (Paramus, $\mathrm{NJ}, \mathrm{USA}$ ). $\mathrm{CI}<1, \mathrm{CI}=1$ and $\mathrm{CI}>1$ indicate synergistic, 
additive and antagonistic effects, respectively.

\section{Cell proliferation assay}

The effect of PIK3R3 siRNA on the proliferation of U-373 MG cells was determined by the trypan blue exclusion assay. For this purpose, the $1 \times 10^{5}$ cells were transfected with $100 \mathrm{nM}$ of PIK3R3 specific and NC siRNAs in 6-well culture plates and incubated for 1-5 days. The cells were then harvested and stained with $0.4 \%$ trypan blue (Merck KGaA, Darmstadt, Germany). After 2 minutes of incubation, the number of viable (unstained) cells was counted using an inverted microscope (Nikon Instrument Inc., Melville, NY, USA) and a hemocytometer. The percentage of viable cells was calculated as follows: cell viability $(\%)=(\mathrm{N}$ Test $/ \mathrm{N}$ blank Control) $\times 100$, where the percentage of viable cell in the control group was considered as $100 \%$.

\section{Apoptosis ELISA assay}

In order to assess the cell death, mono- and oligonucleosomes released into the cytoplasm of apoptotic cells were measured using a cell death detection ELISA plus kit (Roche Diagnostics GmbH). U-373 cells seeded at a density of $1 \times 10^{5}$ cells/well in 6-well plates were treated with $P I K 3 R 3$ siRNA, NC siRNA, the $\mathrm{IC}_{50}$ dose of erlotinib, alone or in combination, as described earlier. At 24 and $48 \mathrm{~h}$ post-transcription, cells were harvested and cell lysates were collected. Following a $10 \mathrm{~min}$ centrifugation of the cell lysates at $200 \mathrm{~g}$, mixtures of $20 \mu \mathrm{l}$ of the supernatants and $80 \mu \mathrm{l}$ of immunoreagent (containing anti-histone-biotin and anti-DNA-peroxidase) were transferred to each well of a streptavidin-coated
Combination Therapy with PIK3R3-siRNA and Erlotinib

plate. After $2 \mathrm{~h}$ incubation at $25^{\circ} \mathrm{C}$, the wells were washed with incubation buffer and then, $100 \mu 1$ of 2, 2-azino-bis (3-ethylbenzthiazoline-6-sulfonic acid) (ABTS) was added to each well. Subsequently, ABTS stop solution was added to terminate the reactions. The optical densities were read at $405 \mathrm{~nm}$ with an ELISA plate reader (with a reference wavelength of $490 \mathrm{~nm}$ )

\section{Statistical analysis}

The results are presented as mean \pm standard deviation (SD) of three experiments. ANOVA and Bonferroni's test were used to determine the statistical significance of differences between groups. A p $<0.05$ was considered significant. Prism 6.1 software was used to analyze all data (GraphPad Software Inc).

\section{Results}

The expression of PIK3R3 $m R N A$ was suppressed by siRNA

The results of RT-qPCR showed that PIK3R3 siRNA significantly decreased the expression of the PIK3R3 mRNA in glioblastoma cells in a time-dependent way (compared to the control group, Figure 1A). After 24, 48 , and $72 \mathrm{~h}$ post-transfection, relative expression levels of PIK3R3 mRNA were $82.10 \%, 72.44 \%$ and $61.02 \%$, respectively. Notably, treatment with NC siRNA had an insignificant effect on the expression of PIK3R3 in comparison with the blank control. Amplification curves were sigmoidal and only one peak was observed in the melting curves (Figure 1B, 1C and 1D).

B)

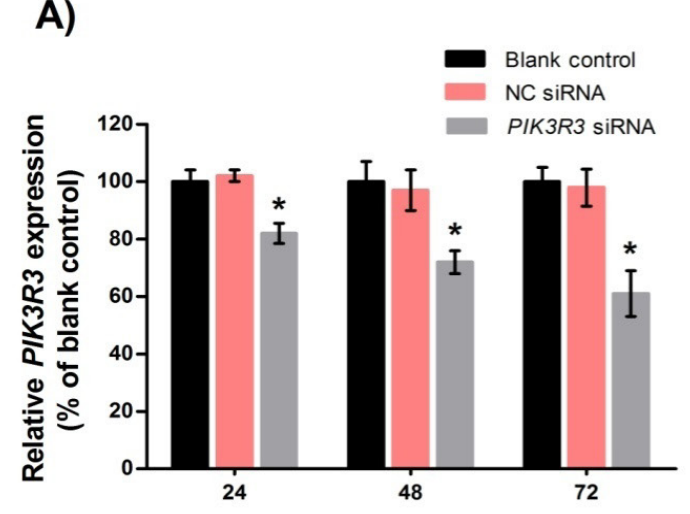

D)

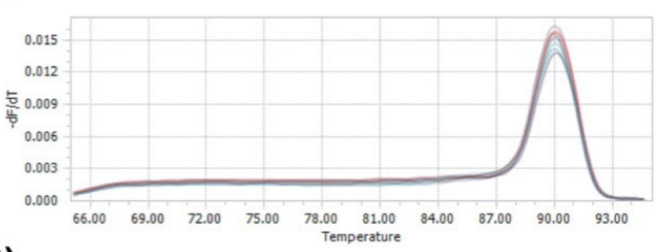

C)

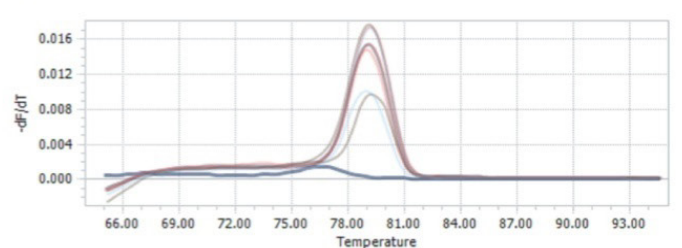

E)

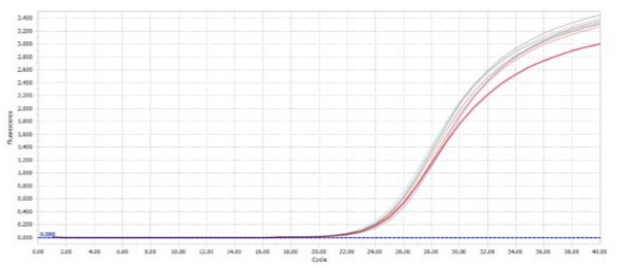

Figure 1. Gene Expression Analysis in U-373 Cells Treated with PIK3R3 siRNA and NC siRNA. The cells were transfected with PIK3R3 siRNA and NC siRNA for 24, 48 and $72 \mathrm{~h}$. Relative $P I K 3 R 3 \mathrm{mRNA}$ expression was calculated using RT-qPCR and $2^{-(\Delta \Delta \mathrm{Ct})}$ method (A). Figure $1 \mathrm{~B}$ and $1 \mathrm{C}$ show the melting curves, and Figure $1 \mathrm{D}$ and $1 \mathrm{E}$ show the proliferation curves of $\beta$-actin and PIK3R3 genes, respectively. The results are showed as mean \pm SD of the results of three experiments. ${ }^{*} p<0.05$ versus corresponding blank control or NC siRNA transfected cells. 
A)

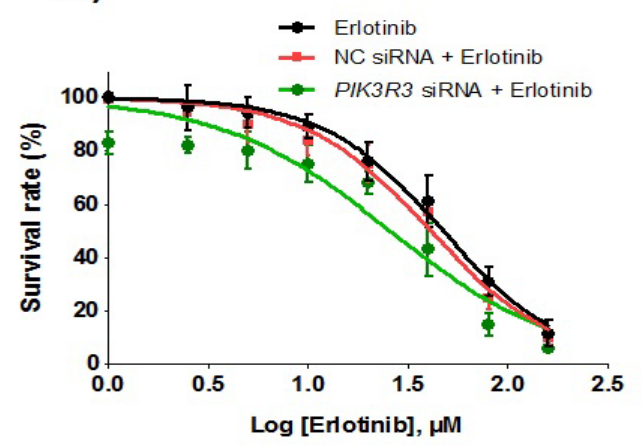

C)

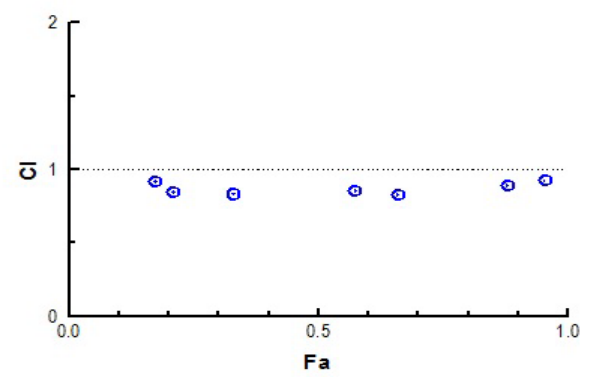

B)

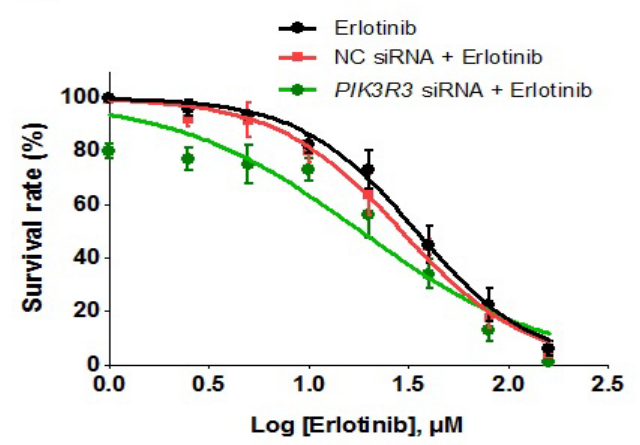

D)

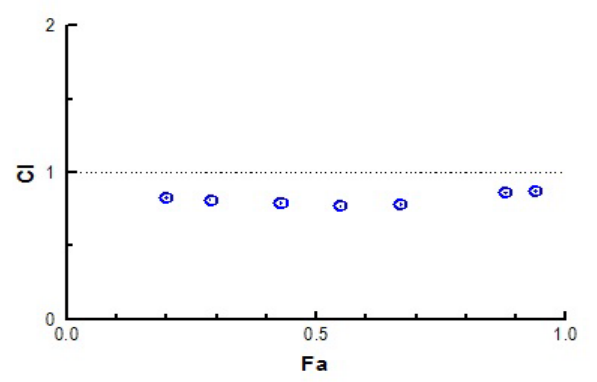

Figure 2. The Effect of PIK3R3 siRNA on Sensitivity of the U-373 Cells to Erlotinib. Cells were treated with PIK3R3 siRNA and different concentrations of erlotinib for $24 \mathrm{~h}$ (A and $\mathrm{C}$ ) and $48 \mathrm{~h}$ (B and D). Next, the cell survival was measured using MTT assay. Cell survival curves (A and B) were plotted using Prism software. The results are presented as mean $\pm \mathrm{SD}(\mathrm{n}=3)$. Data from three independent experiments were used to plot the CI versus $(\mathrm{Fa})(\mathrm{C}$ and D) by the Chou and Talalay method and CalcuSyn software. Dashed lines represent CI=1.

Table 1. $\mathrm{IC}_{50}$ Values of the Erlotinib in Glioblastoma Cells before and after Transfection of siRNAs.

\begin{tabular}{lcc}
\hline Treatment & \multicolumn{2}{c}{$\mathrm{IC}_{50}(\mu \mathrm{M})$} \\
\hline Erlotinib & $44.48 \pm 2.35$ & $31.84 \pm 1.09$ \\
NC siRNA and erlotinib & $41.29 \pm 1.22 \#$ & $28.05 \pm 1.67 \#$ \\
$P I K 3 R 3$ siRNA and erlotinib & $25.86 \pm 1.33^{*}$ & $18.38 \pm 0.84 *$ \\
\hline
\end{tabular}

$\mathrm{IC}_{50}$ values were calculated by sigmoidal dose-response model using Prism software. Data expressed as the mean \pm SD of three independent experiments. ${ }^{*} \mathrm{p}<0.05$ versus corresponding erlotinib; $\# \mathrm{p}>0.05$ relative to the corresponding erlotinib.

PIK3R3 siRNA synergistically enhanced the cytotoxic effect of erlotinib

A combination treatment of erlotinib and PIK3R3 siRNA on U-373 cells was investigated, to determine whether decreased $P I K 3 R 3$ could enhance the sensitivity of the glioblastoma cells to erlotinib. The results of the MTT assay showed monotherapy with erlotinib induced cytotoxicity in a dose-dependent way (Figure 2A, 2B). PIK3R3 siRNA significantly reduced the cell survival rate to $84.61 \%$ and $79.12 \%$ after 24 and $48 \mathrm{~h}$, respectively (compared with the blank control, $\mathrm{p}<0.05$ ). Furthermore, erlotinib in combination with PIK3R3 siRNA further reduced the cell survival rate relative to erlotinib or PIK3R3 siRNA alone $(\mathrm{p}<0.05)$. Moreover, PIK3R3 siRNA markedly reduced the $\mathrm{IC}_{50}$ value of erlotinib from 44.48 $\mu \mathrm{M}$ and $25.86 \mu \mathrm{M}$ to $31.84 \mu \mathrm{M}$ and $18.38 \mu \mathrm{M}$ after 24 and $48 \mathrm{~h}$, respectively (Table 1). Meanwhile, transfection of NC siRNA had a minimal effect on the chemosensitivity of the glioblastoma cells compared with the PIK3R3 siRNA treated cells $(\mathrm{p}>0.05)$.

Combination index analysis showed that combination

Table 2. CI analysis of Erlotinib and PIK3R3 siRNA Combination in U373-MG Glioma Cells

\begin{tabular}{lcccccc}
\hline Erlotinib concentration $(\mu \mathrm{M})$ & \multicolumn{2}{c}{$24 \mathrm{~h}$} & \multicolumn{2}{c}{$48 \mathrm{~h}$} & Combined effect \\
\hline 2.5 & $\mathrm{Fa}$ & $\mathrm{CI}$ & Combined effect & $\mathrm{Fa}$ & $\mathrm{CI}$ & $\mathrm{S}$ \\
5 & 0.175 & 0.91 & $\mathrm{~S}$ & 0.2 & 0.82 & $\mathrm{~S}$ \\
10 & 0.21 & 0.84 & $\mathrm{~S}$ & 0.29 & 0.81 & $\mathrm{~S}$ \\
20 & 0.33 & 0.83 & $\mathrm{~S}$ & 0.43 & 0.79 & $\mathrm{~S}$ \\
40 & 0.575 & 0.85 & $\mathrm{~S}$ & 0.67 & 0.77 & $\mathrm{~S}$ \\
80 & 0.88 & 0.88 & $\mathrm{~S}$ & 0.88 & 0.85 & $\mathrm{~S}$ \\
160 & 0.955 & 0.92 & $\mathrm{~S}$ & 0.94 & 0.87 & $\mathrm{~S}$ \\
\hline
\end{tabular}

The combination index (CI) values were measured with Chou-Talalay method and CompuSyn software. Antagonistic, synergistic (S) and additive effects are defined by $\mathrm{CI}$ value $>1,<1$ and $=1$ or close to 1 , respectively. 


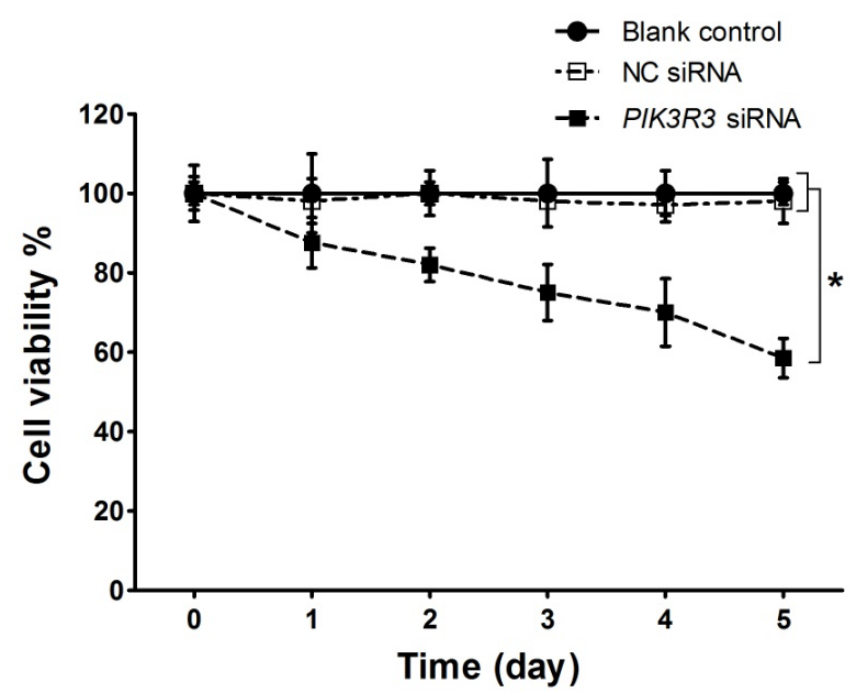

Figure 3. Proliferation Curve of U-373 Cells Treated with PIK3R3 siRNA. Cell proliferation was determined using trypan blue staining over a period of 5 days. Data are expressed as mean $\pm \operatorname{SD}(n=3)$. ${ }^{*} p<0.05$ versus blank control or NC siRNA.

treatment of $P I K 3 R 3$ siRNA and erlotinib was synergistic $(\mathrm{CI}<1)$ (Figure $2 \mathrm{C}$ and $2 \mathrm{D}$ ). The results showed that the best mean CI value of $24 \mathrm{~h}$ were observed at $160 \mu \mathrm{M}$ erlotinib in combination with $P I K 3 R 3$ siRNA $(\mathrm{CI}=0.82)$ with Fa level of 0.96 . Moreover, strongest synergistic effect of $48 \mathrm{~h}(\mathrm{CI}=0.76, \mathrm{Fa}=0.97)$ was occurred at 160 $\mu \mathrm{M}$ of erlotinib (Table 2 ).

Suppression of PIK3R3 expression inhibited the proliferation of $U-373 \mathrm{MG}$ cells

We next sought to examine whether inhibition of $P I K 3 R 3$ could inhibit the growth of cancer cells. The U-373 cells were transfected with PIK3R3 specific siRNA and NC siRNA and then cell viability was assessed using the trypan blue test every $24 \mathrm{~h}$. Results showed that, PIK3R3 siRNA significantly reduced the proliferation of glioblastomas cells, in a time dependent manner $(p<0.05$; Figure 3). Beginning at $24 \mathrm{~h}$ after transfection, the cell

A)

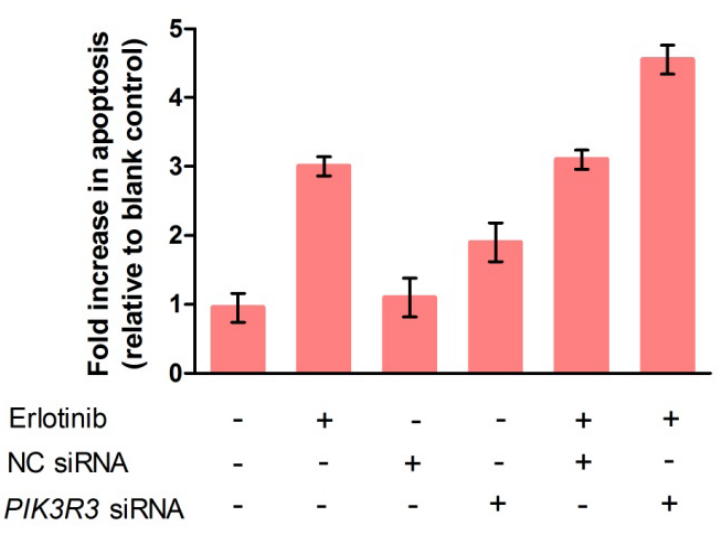

proliferation dropped to $87.10 \%$ and then to a further $56.23 \%$ on day 5 . However, no significant differences in cell proliferation were found between the NC siRNA and blank control groups ( $\mathrm{p}>0.05$; Figure 3 ).

\section{PIK3R3 siRNA augmented the apoptotic effect of erlotinib in U-373 MG cells}

To determine whether the observed synergistic effect of combination treatment was related to the enhancement of apoptosis, the effects of either erlotinib or PIK3R 3 siRNA, alone or in combination, on cell apoptosis was measured by an ELISA cell death assay Kit. Results demonstrated that compared with the blank control group, PIK3R3 siRNA significantly enhanced the extent of apoptosis in a time dependent manner (Figure 4). Moreover, 24 and 48 $\mathrm{h}$ exposure of the cells to erlotinib augmented the extent of apoptosis by 3.11 and 3.78 fold, respectively $(p<0.05)$. Combination treatment further enhanced apoptosis to

\section{B)}

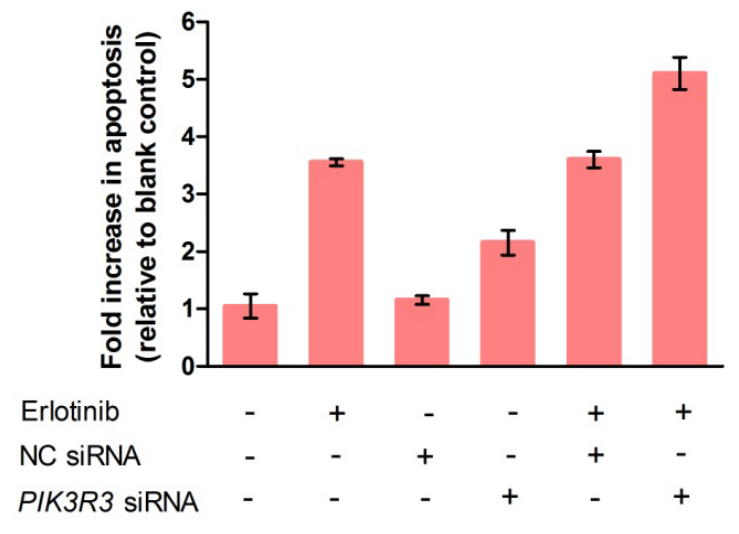

Figure 4. The Effect of Treatments on Apoptosis of Tumor Cells. The U-737 cells were treated with PIK3R3 siRNA (50 $\mathrm{nM})$, negative control (NC) siRNA (50 nM) and erlotinib ( $\mathrm{IC}_{50}$ doses of 24 and $\left.48 \mathrm{~h}\right)$, alone and in combination. The apoptosis was quantified using ELISA cell death assay after 24 (A) and $48 \mathrm{~h}(\mathrm{~B})$. The data are presented mean \pm SD $(n=3)$ of three independent experiments. ${ }^{*} \mathrm{p}<0.05$ compared with control. 
4.64 and 5.39 fold at the indicated time points $(\mathrm{p}<0.05)$. No significant alterations in apoptosis were detected for NC siRNA or NC siRNA plus erlotinib groups relative to the blank control group or erlotinib alone, respectively $(p>0.05)$. Therefore, these results indicate that the chemosensitization effect of $P I K 3 R 3$ siRNA is partially attributed to the induction of apoptosis.

\section{Discussion}

Glioblastoma multiform is the most malignant type of glioma, accounting for more than half of all primary glioma. Although Glioblastoma treatment has progressed in recent years, treatment resistance is high and the overall clinical outcome is still unsatisfactory (Preusser et al., 2008; García-Claver et al., 2013; Alamdari-Palangi et al., 2020a). Thus, developing effective treatments is critical. Erlotinib, a tyrosine kinase inhibitor, is used for treat brain cancers as a second-line medication. Several clinical trials have shown that distinct mechanisms of resistance toward the action of these drugs in glioma cells (Engelman et al., 2006). Knowing the molecular mechanisms responsible for resistance to EGFR inhibitor treatment is essential for developing new combinatorial therapy to enhance response in glioblastoma. In this study, the impact of PIK3R3 specific siRNA on proliferation, apoptosis, and sensitivity in U-373 MG erlotinib resistant human glioblastoma cells is investigated.

PI3K is an intracellular lipid kinase involved in a wide range of cancer-related pathways. Studies indicated that increased PI3K pathway activity might be due to gene replication or increased expression in cancers also it is associated with tumor cell survival and anti-apoptotic properties (Vivanco and Sawyers, 2002; Galanis et al., 2005a). Over-expression or mutations of the PIK3R3 gene, the catalytic subunit of PI3K, have been identified in previous studies (Vivanco and Sawyers, 2002; Tsao et al., 2005; Vredenburgh et al., 2007). SiRNAs are small noncoding RNAs that regulate gene expression (AlamdariPalangi et al., 2020a). Here, we showed that siRNA reduced the expression of $P I K 3 R 3$ mRNA, demonstrating that it is one of the main mechanisms of resistance to erlotinib. Moreover, Suppression of PIK3R3 expression inhibited the proliferation of U-373 cells and increased apoptosis. These findings support $P I K 3 R 3$ as a promising potential therapeutic target for increasing glioblastoma cell responsiveness to erlotinib. The results of earlier research revealed that the expression of PIK3R 3 gene in glioblastoma resistant cells has augmented following exposure to erlotinib that may be one of the primary causes of resistance to this drug (Galanis et al., 2005a). MTT analysis in our study indicated that siRNA treatment of glioblastoma cells increased drug sensitivity. In addition, IC50 value of erlotinib was significantly reduced in the presence of $P I K 3 R 3$ siRNA. Furthermore, treatment of cells with siRNA led to substantial induction of apoptosis and enhanced cancer cell sensitivity to erlotinib-induced apoptosis. A cell proliferation assay was carried out to ascertain the impact of inhibiting PIK3R3 gene expression on glioma cell growth. Due to the suppression of this gene by specific siRNA, cell growth was drastically reduced at predetermined intervals. Our findings support previous research by confirming the importance of the PIK3R3 gene in glioblastoma progression (Shayesteh et al., 1999a; Zhang et al., 2003; Galanis et al., 2005a).

The EGFR is a transmembrane glycoprotein of the ErbB family of RTKs that over-expressed in various types of malignant tumors (Yamaoka et al., 2018). EGFR has been widely implicated in malignant glioma. Approximately $20-50 \%$ of glioblastomas are frequently show high EGFR gene expression and amplification (Raizer et al., 2010). This causes the activation of many signaling pathways including PI3K/Akt and Ras/Raf/ MAPK (Li et al., 2013; Sigismund et al., 2018; Singh and Jadhav, 2018; Jamal et al., 2019). Tumor cell proliferation, invasion, and survival are increased when the PI3K pathway is activated. The studies have shown that glioma cells may have specific mechanisms of resistance to anticancer activity. Further analysis of gene expression in susceptible and resistant cell lines prior to erlotinib therapy reveals that genes involved with the PI3K/AKT pathway are over-expressed in the susceptible cell lines. However, over-expression of these genes occurs in the resistant cell line after treatment (Galanis et al., 2005a). A detailed study of PIK3 pathway genes in glioblastoma cells showed a significant difference between sensitive and resistant cells following EGFR stimulation. It is noteworthy that The $P I K 3 R 3$ gene, which was expressed more in susceptible cells than in resistant cells, was suppressed exclusively in susceptible cells following erlotinib therapy but no reduction in resistant cells was observed. Furthermore, in glioblastoma proliferation, the copy number of the PIK3R3 gene enhanced, which could play a role in cancer cell survival (Shayesteh et al., 1999a; Galanis et al., 2005a). These findings suggest that the PIK3R3 might be an acceptable and interesting therapeutic target for erlotinib responsiveness. In this research, we showed that exposing U-373 MG cells to erlotinib significantly reduced cell survival while increasing apoptosis. Transfection of PIK3R3 specific siRNA significantly reduced PIK3R3 mRNA expression and synergistically enhanced erlotinib cell toxicity. Additionally, SiRNA in combination with erlotinib increased the rate of apoptosis comparison to monotherapy. Our observations support the idea that via blocking the PIK3 pathway could augment the antitumor effects of EGFR-TKIs such as erlotinib in glioblastoma cells.

The results of previous reports are aligned with the findings of our study. For example, Ruano et al. (2008) found that genes associated with the PI3K survival pathway play an essential role in glioblastoma patients and are considered to be diagnostic biomarkers for glioma carcinogenesis. Our results also reveal that over-expression of $P I K 3 R 3$ plays a vital role in glioblastoma chemotherapy resistance, which could be a pioneer therapeutic target for patients. Zhang et al., (2007) showed that enhanced PI3K pathway activity in ovarian cancer may be owing to gene replication or increased PIK3R3 expression. They also examined the function of $P I K 3 R 3$ by specifically suppressing mRNA expression with siRNA and found that inhibition of the $P I K 3 R 3$ mRNA expression enhanced apoptosis in vitro. 
Like our study, they showed that anti-PIK3R3 specific siRNA significantly increased apoptosis and enhanced cellular susceptibility to erlotinib-induced apoptosis. These findings suggest that up-regulation of $P I K 3 R 3$ is linked to the erlotinib-resistance of the glioblastoma cells, and PIK3R3 inhibition could dramatically reduces this resistance.

In conclusion, overall, our results demonstrate that the PIK3R3 gene has a critical role in the growth, survival and drug-resistance of the glioma cells. Treatment with either erlotinib or $P I K 3 R 3$ siRNA markedly triggers apoptosis and inhibits the proliferation of the glioblastoma cells. Moreover, combining PIK3R3 siRNA with erlotinib improves the therapeutic efficacy of the erlotinib. Therefore, $P I K 3 R 3$ could be an effective therapeutic target to reverse erlotinib-resistance of the glioblastoma patients.

\section{Author Contribution Statement}

Study concept and design: HK; Acquisition of data: RA and MB; Analysis and interpretation of data: HK, MB and RA; Drafting of the manuscript: RA and HK; Critical revision of the manuscript for important intellectual content: HK and MB; Funding recipients: HK.

\section{Acknowledgments}

This work is part of M.Sc thesis of Razieh Amini that was supported by a grant from the Faculty of Medicine, Arak University of Medical Sciences, Arak, Iran.

\section{Funding Statement}

This work was supported by a grant from the Faculty of Medicine, Arak University of Medical Sciences, Arak, Iran [Grant number 1170].

\section{Ethical approval}

This research was ethically wise approved from Deputy of research and technology, Arak University of Medical Sciences, Arak, Iran [Number 1170].

\section{Conflict of interest}

The authors have no conflict of interest to declare

\section{References}

Alamdari-Palangi V, Amini R, Karami H (2020a). MiRNA-7 enhances erlotinib sensitivity of glioblastoma cells by blocking the IRS-1 and IRS-2 expression. J Pharm Pharmacol, 72, 531-8.

Alamdari-Palangi V, Karami Z, Karami H, et al (2020b). MiRNA-7 replacement effect on proliferation and tarcevasensitivity in U373-MG cell line. Asian Pac J Cancer Prev, 21, 1747-53.

Amri J, Molaee N, Baazm M, et al (2019a). Targeting epidermal growth factor receptor by MiRNA-145 inhibits cell growth and sensitizes NSCLC cells to erlotinib. Asian Pac J Cancer Prev, 20, 2781-7.

Amri J, Molaee N, Karami H (2019b). Up-regulation of MiRNA$125 \mathrm{a}-5 \mathrm{p}$ inhibits cell proliferation and increases EGFR-TKI induced apoptosis in lung cancer cells. Asian Pac J Cancer Prev, 20, 3361-7.
Amri J, Molaee N, Karami H, et al (2021). Combination of two miRNAs has a stronger effect on stimulating apoptosis, inhibiting cell growth, and increasing erlotinib sensitivity relative to single miRNA in A549 lung cancer cells. Biotechnol Appl Biochem, 67, 1-12.

Ashofteh N, Amini R, Molaee N, et al (2021). MiRNA-mediated knock-down of Bcl-2 and Mcl-1 increases fludarabinesensitivity in CLL-CII cells. Asian Pac J Cancer Prev, 22, 2191-8.

Bader A, Brown D, Stoudemire J, et al (2011). Developing therapeutic microRNAs for cancer. Gene Ther, 18, 1121-6.

Engelman JA, Luo J, Cantley LC (2006). The evolution of phosphatidylinositol 3-kinases as regulators of growth and metabolism. Nature reviews. Genetics, 7, 606.

Galanis E, Buckner JC, Maurer MJ, et al (2005a). Phase II trial of temsirolimus (CCI-779) in recurrent glioblastoma multiforme: a North Central Cancer Treatment Group Study. J Clin Oncol, 23, 5294-304.

Galanis E, Buckner JC, Maurer MJ, et al (2005b). Phase II trial of temsirolimus (CCI-779) in recurrent glioblastoma multiforme: a North Central Cancer Treatment Group Study. J Clin Oncol, 23, 5294-304.

García-Claver A, Lorente M, Mur P, et al (2013). Gene expression changes associated with erlotinib response in glioma cell lines. Eur J Cancer, 49, 1641-53.

Halatsch M-E, Löw S, Mursch K, et al (2009). Candidate genes for sensitivity and resistance of human glioblastoma multiforme cell lines to erlotinib. J Neurosurg, 111, 211-8.

Jamal J, Molaee N, Karami H (2019). Up-regulation of MiRNA$125 \mathrm{a}-5 \mathrm{p}$ inhibits cell proliferation and increases EGFR-TKI induced apoptosis in lung cancer cells. Asian Pac J Cancer Prev, 20, 3361.

Karami H, Baradaran B, Esfahani A, et al (2013). siRNAmediated silencing of survivin inhibits proliferation and enhances etoposide chemosensitivity in acute myeloid leukemia cells. Asian Pac J Cancer Prev, 14, 7719-24.

Karami H, Baradaran B, Esfehani A, et al (2014). Downregulation of Mcl-1 by small interference RNA induces apoptosis and sensitizes HL-60 leukemia cells to etoposide. Asian Pac J Cancer Prev, 15, 629-35.

Katso R, Okkenhaug K, Ahmadi K, et al (2001). Cellular function of phosphoinositide 3-kinases: implications for development, immunity, homeostasis, and cancer. Ann Rev Cell Develop Biol, 17, 615-75.

Kreisl TN, Kim L, Moore K, et al (2009). Phase II trial of single-agent bevacizumab followed by bevacizumab plus irinotecan at tumor progression in recurrent glioblastoma. J Clin Oncol, 27, 740.

Li M, Li J, Liu L, et al (2013). MicroRNA in human glioma. Cancers, 5, 1306-31.

Luo J, Manning BD, Cantley LC (2003). Targeting the PI3K-Akt pathway in human cancer: rationale and promise. Cancer Cell, 4, 257-62.

Mellinghoff IK, Wang MY, Vivanco I, et al (2005). Molecular determinants of the response of glioblastomas to EGFR kinase inhibitors. N Engl J Med, 353, 2012-24.

Oprita A, Baloi SC, Staicu GA, et al (2021). Updated insights on EGFR signaling pathways in glioma. Int $\mathrm{J} \mathrm{Mol} \mathrm{Sci,} 22$.

Parsons R (2005). Phosphatidylinositol 3-kinase inhibitors are a triple threat to ovarian cancer. Clin Cancer Res, 11, 7965-6.

Paul I, Bhattacharya S, Chatterjee A, et al (2013). Current understanding on EGFR and Wnt/ $\beta$-Catenin signaling in glioma and their possible crosstalk. Genes Cancer, 4, 427-46.

Pirayesh Islamian J, Mohammadi M, Baradaran B, et al (2016). Enhancing radiosensitivity of TE1, TE8, and TE 11 esophageal squamous carcinoma cell lines by Hdm2-siRNA targeted gene therapy in vitro. Bioimpacts, 6, 93-8. 
Preusser M, Gelpi E, Rottenfusser A, et al (2008). Epithelial growth factor receptor inhibitors for treatment of recurrent or progressive high grade glioma: an exploratory study. J Neurooncol, 89, 211-8.

Raizer JJ, Abrey LE, Lassman AB, et al (2010). A phase I trial of erlotinib in patients with nonprogressive glioblastoma multiforme postradiation therapy, and recurrent malignant gliomas and meningiomas. Neurooncology, 12, 87-94.

Ruano Y, Mollejo M, Camacho FI, et al (2008). Identification of survival-related genes of the phosphatidylinositol 3'-kinase signaling pathway in glioblastoma multiforme. Cancer, 112, $1575-84$.

Shahverdi M, Amini R, Amri J, et al (2020). Gene therapy with MiRNA-mediated targeting of Mcl-1 promotes the sensitivity of non-small cell lung cancer cells to treatment with ABT-737. Asian Pac J Cancer Prev, 21, 675-81.

Shahverdi M, Amri J, Karami H, et al (2021). Knockdown of myeloid cell leukemia-1 by MicroRNA-101 increases sensitivity of A549 lung cancer cells to etoposide. Iran J Med Sci, 46, 298-307.

Shayesteh L, Lu Y, Kuo W-L, et al (1999a). PIK3CA is implicated as an oncogene in ovarian cancer. Nat Genet, 21.

Shayesteh L, Lu Y, Kuo WL, et al (1999b). PIK3CA is implicated as an oncogene in ovarian cancer. Nat Genet, 21, 99-102.

Sigismund S, Avanzato D, Lanzetti L (2018). Emerging functions of the EGFR in cancer. Mol Oncol, 12, 3-20.

Singh M, Jadhav HR (2018). Targeting non-small cell lung cancer with small-molecule EGFR tyrosine kinase inhibitors. Drug Discovery Today, 23, 745-53.

Soroceanu L, Kharbanda S, Chen R, et al (2007). Identification of IGF2 signaling through phosphoinositide-3-kinase regulatory subunit 3 as a growth-promoting axis in glioblastoma. Proc Nat Acad Sci U S A, 104, 3466-71.

Tsao M-S, Sakurada A, Cutz J-C, et al (2005). Erlotinib in lung cancer-molecular and clinical predictors of outcome. N Engl J Med, 353, 133-44.

Vivanco I, Sawyers CL (2002). The phosphatidylinositol 3-kinase-AKT pathway in human cancer. Nat Rev Cancer, 2, 489-501.

Vredenburgh JJ, Desjardins A, Herndon JE, et al (2007). Bevacizumab plus irinotecan in recurrent glioblastoma multiforme. J Clin Oncol, 25, 4722-9.

Yamaoka T, Kusumoto S, Ando K, et al (2018). Receptor tyrosine kinase-targeted cancer therapy. Int J Mol Sci, 19, 3491.

Zhang L, Huang J, Yang N, et al (2007). Integrative genomic analysis of phosphatidylinositol 3'-kinase family identifies PIK3R3 as a potential therapeutic target in epithelial ovarian cancer. Clin Cancer Res, 13, 5314-21.

Zhang L, Yang N, Katsaros D, et al (2003). The oncogene phosphatidylinositol 3'-kinase catalytic subunit $\alpha$ promotes angiogenesis via vascular endothelial growth factor in ovarian carcinoma. Cancer Res, 63, 4225-31.

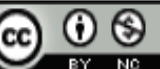

This work is licensed under a Creative Commons AttributionNon Commercial 4.0 International License. 